Afrika Focus, Vol. 11, N 1-4, 1995, pp.145-148

\title{
SITUATION ACTUELLE DE LA TRYPANOSOMIASE EN UBANGI ET PROBLEMES INHERENTS A LA LUTTE CONTRE CETTE MALADIE
}

\section{SEMA HURUGO}

\section{SITUATION}

La maladie du sommeil est actuellement un problème très préoccupant en Ubangi.

Elle sévit dans la plupart des zones de santé dont les plus touchées sont:

- Zones de Santé de Karawa et Loko dans le Nord-Ubangi

- Zones de Santé de Gemena, Bwamanda et Tandala dans la Sous-Région du Sud-Ubangi.

On signale également des cas dans l'aire du centre de santé de référence de Yakamba dans la zone de santé de Budjala.

En 1993, 3.961 trypanosés ont été dépistés.

En 1994, avec des ressources très limitées, nous avons pu dépister (de décembre '93 à septembre '94) 5.985 trypanosés.

Les données actuellement disponibles se présentent de la manière suivante: 
* Population totale examinée par zone de santé

\begin{tabular}{||l|l|l|l||}
\hline ZONE DE SANTE & P.T.E. en D.A. & P.T.E. en D.P. & TOTAL \\
\hline 1. Gemena & 11.535 & 1.419 & 12.954 \\
\hline 2. Bwamanda & 17.514 & 1.823 & 19.337 \\
\hline 3. Karawa & 33.792 & 4.779 & 38.591 \\
\hline 4. Tandala & 44.861 & 175 & 45.036 \\
\hline 5. Bomboma & 968 & 7 & 975 \\
\hline 6. Loko & - & 167 & 167 \\
\hline TOTAL & 108.670 & 8.390 & 117.060 \\
\hline
\end{tabular}

* Répartition de nouveaux cas selon les circonstances de dépistage et stade de la maladie par zone de santé

\begin{tabular}{|c|c|c|c|c|c|c|c|}
\hline \multirow{2}{*}{$\begin{array}{l}\text { ZONE DE } \\
\text { SANTE }\end{array}$} & \multicolumn{3}{|c|}{ D.A. } & \multicolumn{3}{|c|}{ D.P. } & \multirow{2}{*}{$\begin{array}{c}\text { TO- } \\
\text { TAL }\end{array}$} \\
\hline & $\begin{array}{l}1 S \\
T\end{array}$ & $2 S T$ & INC & $\begin{array}{l}1 S \\
T\end{array}$ & $2 S T$ & INC & \\
\hline 1. Gemena & 104 & 473 & 2 & 95 & 961 & - & 1.635 \\
\hline 2. Bwamanda & 47 & 57 & 1 & 135 & 589 & - & 829 \\
\hline 3. Karawa & 107 & 517 & 45 & 305 & 1.823 & 15 & 2.812 \\
\hline 4. Tandala & 124 & 240 & 10 & 34 & 176 & 2 & 586 \\
\hline 5. Bomboma & 1 & 18 & - & 1 & 2 & - & 22 \\
\hline 6. Loko & - & - & - & 10 & 91 & - & 101 \\
\hline TOTAL & 383 & 1.305 & 58 & 580 & 3.642 & 17 & 5.985 \\
\hline
\end{tabular}




\section{STRUCTURES ACTUELLES DE LUTTE CONTRE LA TRYPANOSOMIASE EN UBANGI}

La coordination de la lutte contre la maladie de sommeil est assurée par le C.D.I. Bwamanda, ASBL.

Deux équipes mobiles sont opérationnelles dont la responsabilité a été répartie en 2 zones d'activités:

- la zone d'activités du Nord-Ubangi (zones de santé de Karawa et Loko) avec l'Unité Mobile de Boyasebego.

- la zone d'activités du Sud-Ubangi (zones de santé Gemena, Bwamanda et Tandala) avec l'Unité Mobile de Bwamanda.

Les zones de santé: certaines zones de santé ont intégré la lutte contre la maladie du sommeil dans leurs activités de soins de santé primaires avec toutes les insuffisances dues au manque de compétence. Il va sans dire qu'il n'y avait pas de solutions de rechange.

\section{PROBLEMES}

Insuffisance de la couverture de la population à risque par le dépistage actif. Il y a actuellement 2 équipes mobiles pour une population à risque estimée à peu près à 1 million habitants.

Rupture fréquente en trypanocides entraînant une démobilisation de la population pour les recensements et une démotivation du personnel médical.

Manque de moyens logistiques pour la coordination et les unités mobiles (véhicules, matériel de campement, etc....). La coordination n'a pas de véhicule et les 2 équipes ont encore deux vieux véhicules souvent en panne.

Manque de compétence en matière de trypanosomiase du personnel médical dans les aires de santé à problème trypanosomiase ainsi que dans les hôpitaux généraux de référence. Manque de pièges pour la lutte antivectorielle. 


\section{SUGGESTIONS}

Améliorer la couverture de la population à risque en créant au moins 4 équipes mobiles supplémentaires (pour les Zones de Santé de Karawa, Gemena, Tandala et Budjala) et les doter de moyens suffisants.

Assurer un approvisionnement régulier en trypanocides.

Les besoins mensuels en trypanocides:

- Arsobal: $\quad 5.500$ ampoules

- Bayer 205: 300 flacons

- Pentamidine: 500 flacons

- D.F.M.O. et/ou Lampit pour des cas Arsobal résistants (30 malades par mois)

Doter la coordination et les équipes mobiles de nouveaux véhicules tout terrain et assurer leur fonctionnement.

Assurer une formation à toutes les catégories des professionnels de la santé en trypanosomiase. Un seminaire, à l'intention des médecins et infirmiers oeuvrant dans les 2 Sous-Régions, s'avère nécessaire au début de l'année prochaine (1995).

Fournir des pièges pour la lutte antivectorielle après avoir formé les piégeurs.

\section{CONCLUSIONS}

De toute urgence nous faisons appel aux institutions nationales, pour qu'elles remédient à la situation alarmante de la maladie du sommeil en Ubangi.

Nous sollicitons un appui auprès des institutions internationales pour pouvoir contrôler cette endémie qui bientôt aura des répercussions sur le développement de la région. 